\title{
Oocyte cryopreservation review: outcomes of medical oocyte cryopreservation and planned oocyte cryopreservation
}

\author{
Zachary Walker ${ }^{*} \mathbb{D}$, Andrea Lanes and Elizabeth Ginsburg
}

\begin{abstract}
Background: The utilization of oocyte cryopreservation (OC) has become popularized with increasing numbers of reproductive-aged patients desiring to maintain fertility for future family building. OC was initially used for fertility preservation in postmenarchal patients prior to gonadotoxic therapies; however, it is now available to patients to circumvent age-related infertility and other diagnoses associated with early loss of ovarian reserve. The primary aim of this paper is to provide a narrative review of the most recent and robust data on the utilization and outcomes of OC in both patient populations.

Summary: OC results in similar oocyte yield in patients facing gonadotoxic therapies and patients undergoing planned OC. Available data are insufficient to predict the live birth rates or the number of oocytes needed to result in live birth. However, oocyte yield and live birth rates are best among patients $<37.5$ years old or with anti-mullerian hormone levels $>1.995 \mathrm{ng} / \mathrm{dL}$, at the time of oocyte retrieval. There is a high 'no use' rate (58.9\%) in patients using planned OC with $62.5 \%$ returning to use frozen oocytes with a spouse. The utilization rate in medical OC patients is $<10 \%$. There is currently no data on the effects of BMI, smoking, or ethnicity on planned OC outcomes.

Conclusion: It is too early to draw any final conclusions on outcomes of $\mathrm{OC}$ in medical $\mathrm{OC}$ and planned OC; however, preliminary data supports that utilization of $\mathrm{OC}$ in both groups result in preservation of fertility and subsequent live births in patients who return to use their cryopreserved eggs. Higher oocyte yield, with fewer ovarian stimulation cycles, and higher live birth rates are seen in patients who seek $\mathrm{OC}$ at younger ages, reinforcing the importance of age on fertility preservation. More studies are needed in medical $O C$ and planned $O C$ to help guide counseling and decision-making in patients seeking these services.
\end{abstract}

Keywords: Cost effectiveness, Fertility preservation, Oocyte freezing, Oocyte warm, Oocyte utilization, Planned oocyte cryopreservation, Vitrification

\section{Background}

Oocyte cryopreservation $(\mathrm{OC})$ is used in assisted reproductive technology (ART) to attempt to preserve fertility by freezing gametes for potential future use. The first human pregnancy (twins) from a previously

*Correspondence: zwalker1@bwh.harvard.edu Department of Obstetrics and Gynecology, Division of Reproductive Endocrinology and Infertility, Brigham \&Women's Hospital, 75 Francis Street, Boston, MA 02115, USA cryopreserved oocyte was reported in 1986 [1]. With improved techniques of cryopreservation (i.e., slowfreeze to ultra-rapid vitrification) there have been tremendous improvement in oocyte survival and clinical pregnancy rates [2, 3]. In 2013 the American Society for Reproductive Medicine (ASRM) removed the "experimental" label associated with OC. The American College of Obstetricians and Gynecologist (ACOG) promoted the utilization of OC in 2014 [4]. At that time, a majority of $\mathrm{OC}$ was used for patients with upcoming exposure to original author(s) and the source, provide a link to the Creative Commons licence, and indicate if changes were made. The images or other third party material in this article are included in the article's Creative Commons licence, unless indicated otherwise in a credit line to the material. If material is not included in the article's Creative Commons licence and your intended use is not permitted by statutory regulation or exceeds the permitted use, you will need to obtain permission directly from the copyright holder. To view a copy of this licence, visit http://creativecommons.org/licenses/by/4.0/. The Creative Commons Public Domain Dedication waiver (http://creativeco mmons.org/publicdomain/zero/1.0/) applies to the data made available in this article, unless otherwise stated in a credit line to the data. 
gonadotoxic therapies including chemotherapy and pelvic radiation, and genetic disorders predisposing them to primary ovarian insufficiency (e.g., Fragile $\mathrm{X}$ premutation and monosomy X mosaicism). A $25 \%$ increase in the utilization of OC was seen from 2015 to 2016 [5]. In 2018 the ASRM Ethics Committee opinion stated that planned OC for patients wishing to attempt to protect against future infertility due to reproductive aging was 'ethically permissible' [6]. ASRM continues to recommend that providers inform patients about the efficacy, safety, benefits and risks, as well as unknown long-term effects on offspring and potential harms that are still not fully understood [6].

Oocyte cryopreservation has increased worldwide [7]. However, reproductive age patients are still inadequately aware of the effects of age on fertility [8-12]. Most patients seeking OC obtain information from online sources [13]. Interestingly, a study of obstetricians and gynecologists revealed that, although they believe discussions of reproductive aging should be discussed with all reproductive aged patients, a majority reported a lack of time or knowledge to counsel patients on fertility preservation [14]. Some opinions hold that patients are "delaying" childbearing due to career aspirations; however, this is actually not the case. Most patients who pursue OC report that the reason is the lack of a suitable partner and a source of protection against future medical issues that may affect fertility $[8,15-17]$. It is imperative that healthcare providers be equipped with the knowledge and information to counsel patients regarding reproductive aging and the option of OC.

The purpose of this narrative review on medical OC and planned $\mathrm{OC}$ is to compare the most recent available data on outcomes, expectations, and overall recommendations to help guide decision-making and clinical counseling for patients.

\section{Materials and methods}

\section{Search strategy and study selection}

We searched the published articles in PubMed that contained the key words "autologous IVF", "fertility preservation", "oocyte cryopreservation", "elective", "cancer", "medical", or "planned". We did not include abstracts, conference proceedings, review articles, or case-reports. Studies that included pediatric, adolescent, or transgender patients were excluded. We included randomized controlled trials, systematic reviews or meta-analyses, cohort studies, and case control studies that had full-length manuscripts that were published in English in peer-reviewed journals up to July 2021. We also included studies focusing on medical OC, planned OC, and simulated/hypothetical patient cohorts if part of a cost-effectiveness study. Animal studies, studies without a comparison group (unless addressing topics of utilization of oocytes), inclusion of donor oocytes, utilizing slow-cooling techniques only, articles pertaining to surgical interventions (e.g., ovarian tissue cryopreservation or fertility-sparing surgery), in vitro maturation, frozen embryos only, and studies focused on laboratory-related technical issues were excluded. We screened the titles and abstracts of potentially related and relevant articles based on the above inclusion/exclusion criteria. We included all articles that pertained to utilization of medical OC and/ or planned OC. Final inclusion or exclusion decisions were based on examination of the articles in full.

The following data were recorded: author, year of publication, study design, sample size (patients and/ or cycles), stimulation protocol (if stated), mean age at cryopreservation, mean number of oocytes retrieved, mean number of mature oocytes (MIIs) cryopreserved, return/utilization rate, fertilization rate of MIIs, storage duration, clinical pregnancy rate, and ongoing pregnancy/live birth rate.

\section{Medical oocyte cryopreservation}

Initially, OC was primarily used in patients who were going to be exposed to gonadotoxic therapies or radiation for treatment of malignancies. However, indications have expanded, such as preserving supernumerary oocytes for future use in situations where fertilizing all oocytes is not desired, unexpected unavailability of sperm at the time of oocyte retrieval, and planned female-to-male transition [18]. For the purposes of this review, we primarily focused on medical OC that pertained to patients who were diagnosed with cancer and were expected to undergo gonadotoxic therapy.

There have been significant improvements in the management of cancers leading to increased survival rates. Fertility preservation has become integral to the discussion of cancer treatment in reproductive age patients who are at risk of infertility after therapy. One study found that approximately $70 \%$ of young female cancer patients were concerned about fertility at the time of diagnosis, and $50 \%$ desired to have children after treatment [19]. The risk of premature ovarian failure or diminished ovarian reserve after cancer treatment is dependent on age, chemotherapy used, dosage of chemotherapy agents, and exposure to pelvic radiation. It is of the utmost priority that oncologists speak with their patients about their risks of infertility and to review potential options for fertility preservation prior to initiation of treatment, or refer them for this counseling. 


\section{Outcomes of medical oocyte cryopreservation}

There are limited data regarding outcomes of autologous $\mathrm{OC}$ in patients with cancer (Table 1). Cobo et al. published a retrospective, observational multicenter study that included patients who underwent planned OC and medical OC from January 2007 to May 2018. They included 1073 patients (1172 cycles) who underwent medical OC. The mean age was $32.3 \pm 3.5$ years old with $69.9 \%$ of patients being $\leq 35$ years old. They primarily used an antagonist plus letrozole protocol for controlled ovarian stimulation; however, they noticed that the antagonist only protocol yielded higher number of retrieved and vitrified oocytes compared to the long GnRH-agonist and the antagonist plus letrozole groups. The mean number of mature oocytes retrieved and vitrified per cycle was $11.4 \pm 3.5$ and $8.7 \pm 2.1$, respectively. Only $80(7.4 \%)$ patients returned to used their cryopreserved oocytes with a mean storage time of $4.1 \pm 0.9$ years. The embryo transfers were done on either day 3 or at blastocyst stage (days 5-7). The overall survival rate and implantation rate was 81.8 and 32.5\%, respectively. The clinical pregnancy rate per transfer was $41.4 \%$ and the ongoing pregnancy rate per transfer was $31.0 \%$. There were 18 live births within their cohort of medical OC. When factoring in age, they did not notice a statistically significant difference in number of oocytes needed to have a live birth; however, this was most likely due to small sample size. When compared to patients who underwent planned $\mathrm{OC}$, medical $\mathrm{OC}$ patients were younger at the age of vitrification, had higher numbers of oocytes retrieved and vitrified per cycle, longer storage times, lower implantation rates, ongoing pregnancy rates, and live birth rates $(p<0.05)$.

Two retrospective studies by Moraes et al. and Schon et al. also compared medical OC to planned OC $[22,25]$. Moraes et al. included 23 cancer patients (primarily diagnosed with breast cancer) and 164 non-cancer patients undergoing medical $\mathrm{OC}$ and planned $\mathrm{OC}$, respectively. The mean age was $35.13 \pm 3.72$ years old. Contrary to Cobo et al., Moraes et al. found no statistically significant difference in mean number of oocytes obtained per cycles (11.4 \pm 8 non-cancer vs. $13.8 \pm 9$ cancer) and mean number of frozen mature oocytes per cycle $(9.7 \pm 7$ noncancer vs. $11.2 \pm 7.2$ cancer). However, the inability to find a statistically significant difference may have been due to the small sample size.

Schon et al. included 117 patients ( 130 cycles) undergoing medical OC to 98 patients (129cycles) undergoing planned OC. Stimulation protocols used included GnRH agonist, GnRH antagonist, or flare. Patients undergoing medical OC were more likely to use an antagonist protocol compared to patients undergoing planned OC $(92.9 \%$ vs. $77.8 \%, p=0.003)$. When adjusting for age, there were no statistically significant differences in cycle parameters (e.g., gonadotropin dosage, estradiol level, number of follicles measuring $>15 \mathrm{~mm}$ at time of trigger) between medical OC and planned OC. There was no difference in number of mature oocytes retrieved (7 (range, 4-13) planned OC vs. 8 (range, 6-15) medical OC, $p=0.23$ ).

Another study by Specchia et al. [26] investigated 18 years of experience of medical OC at a tertiary care referral center. They included all 244 patients who underwent 252 cycles of medical OC from January 2001 to March 2019 at a single-center. A majority of the patients were diagnosed with breast cancer (59.9\%) or Hodgkin's or non-Hodgkin's lymphoma (27.4\%). The primary stimulation protocol was an antagonist protocol; however, patients received antagonist plus an aromatase inhibitor if they had hormone-dependent breast cancers. The mean age of the cohort was $31.3 \pm 6.4$ years, which is similar to that of Cobo et al. [7]. The mean number of oocytes retrieved and mature oocytes vitrified per patient were $13.5 \pm 8.4$ (range, 0-40) and 9.5 \pm 6.1 (range, 0-28), respectively. The return rate for use of cryopreserved oocytes was $4.5 \%$ with a short mean duration of storage of 3.4 years showing that as of the time of publication a majority of patients who undergo medical OC had not yet returned to utilize their vitrified oocytes. The reasons for this were not investigated, but may have included cancer progression requiring further treatment, spontaneous pregnancy, or lack of a partner. As tamoxifen use for 10 years is recommended for patients with ER positive breast cancer, some delays may have been due to wanting to maximize disease free survival. The clinical pregnancy rate of the 11 patients who returned to use their cryopreserved oocytes was $36.4 \%$ per patient (16.7\% per transfer) with a total of 2 live births overall. At the conclusion of the study, $95.7 \%$ of oocytes retrieved were still in storage.

A prospective study by Marklund et al. investigated the efficacy and safety of controlled ovarian stimulation protocols using GnRH antagonist with or without the addition of letrozole in breast cancer patients undergoing medical $\mathrm{OC}$ [21]. This was a prospective, multicenter study from January 1, 1995 to June 30, 2017 at six Swedish fertility programs. Approximately 600 women were enrolled with 468 undergoing fertility preservation. Forty-one percent of those were undergoing medical OC. The mean age of the overall cohort was 32.5 years old (range, 21-42). Marklund et al. compared GnRH antagonist with letrozole $(n=224)$ with $\mathrm{GnRH}$ antagonist without letrozole $(n=156)$, conventional start $(n=179)$ versus random start $(n=201)$, and the use of GnRH agonist trigger $(n=96)$ versus hCG trigger $(n=128)$ in patients using a GnRH antagonist protocol with letrozole. They found no statistically significant differences in number of cryopreserved oocytes when using 


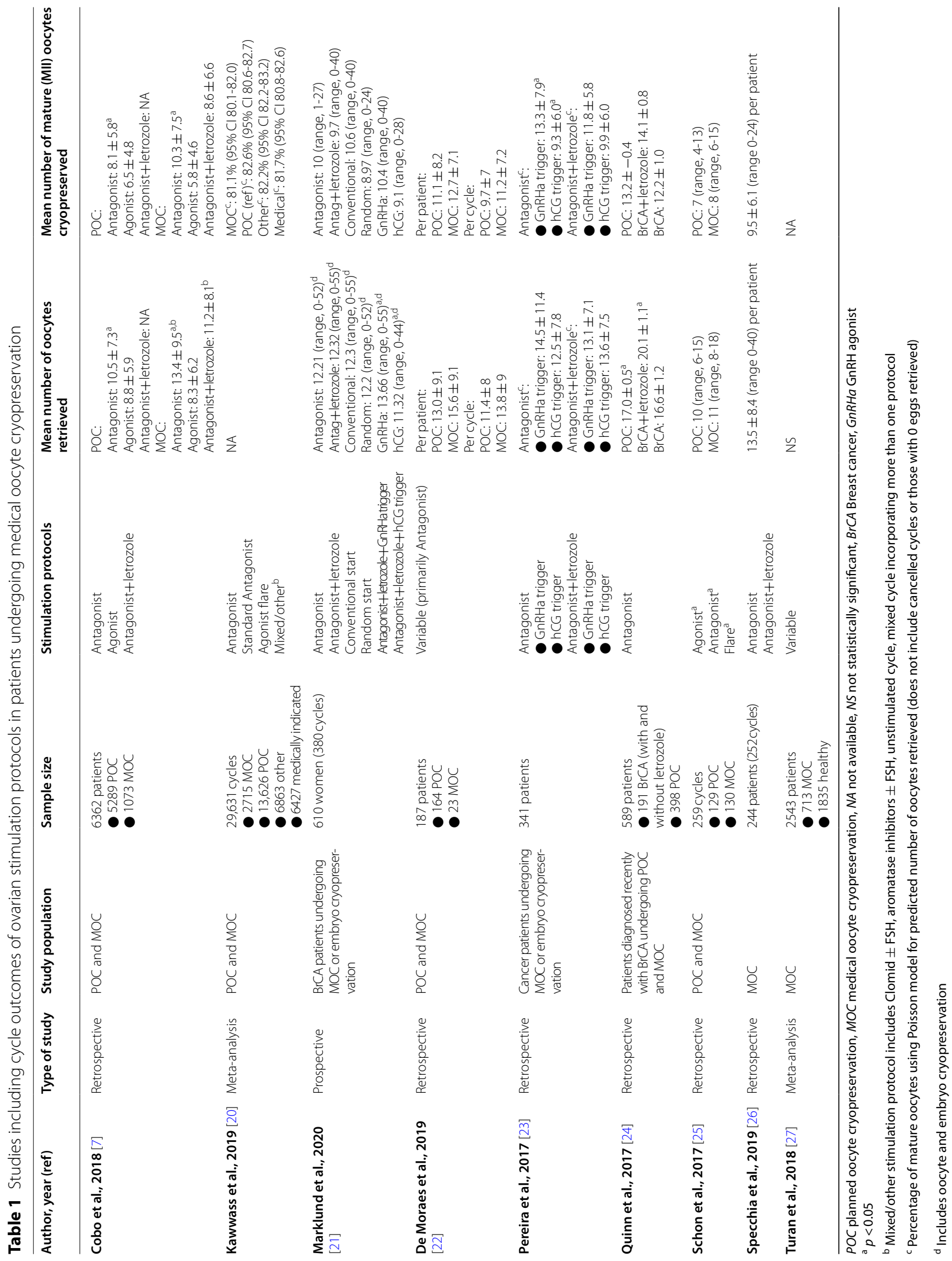


$\mathrm{GnRH}$ antagonist with letrozole versus without letrozole (mean number of cryopreserved oocytes: 9.7 with letrozole vs 10 without letrozole, $p=0.81$ ) or between conventional start versus random start (mean number of cryopreserved oocytes: 10.6 conventional vs 8.97 random, $p=0.067)$. However, in patients utilizing $\mathrm{GnRH}$ antagonist with letrozole and $\mathrm{GnRH}$ agonist trigger had a higher number of oocytes retrieved compared to patients with $\mathrm{GnRH}$ antagonist with letrozole and hCG trigger (13.66 GnRH agonist trigger vs. 11.32 hCG trigger, $p=0.027)$. This was also seen in a study by Pereira et al. [23] who investigated cancer patients undergoing controlled ovarian stimulation for fertility preservation with GnRH antagonist protocol with or without letrozole and compared cycle outcomes with either use of a $\mathrm{GnRH}$ agonist trigger versus hCG trigger. They included 341 patients with an overall mean age of $33.3 \pm 5.1$ years. The primary cancer diagnosis was breast cancer (75.3\%) followed by lymphoma/leukemia (9.7\%). They found that using a GnRH agonist trigger resulted in higher number of MII oocytes cryopreserved compared to hCG trigger in patients using letrozole-based protocols $(11.8 \pm 5.8$ GnRH agonist vs. 9.9 $\pm 6.0 \mathrm{hCG}, p=0.04)$ and those using gonadotropin-only protocols $(13.3 \pm 7.9 \mathrm{GnRH}$ agonist vs. $9.3 \pm 6.0, p=0.02$ ).

Kawwass et al. [20] conducted a national retrospective review using national surveillance data on 29,631 autologous OC cycles from the Society for Assisted Reproductive Technology Clinical Outcomes Reporting System (SART CORS) performed from 2012 to 2016 to compare outcomes of fertility preservation between patients with and without cancer. When compared to planned OC, patients undergoing medical OC were typically $<35$ years old, had higher body mass indices (BMI), lived in the South, and underwent antagonist protocols. There was no difference in cancellation or hyperstimulation rates, or oocyte yield (approximately 16 oocytes, $80 \%$ maturation rate) between the two groups, as also shown in prior studies [24, 27]. Neither oocyte fertilization rates nor live birth rates were reported.

Lyttle Schumacher et al. did a cost-effectiveness study on OC for cancer patients prior to high and low-risk gonadotoxic therapy [28]. The purpose of their study was to find the live birth rate and cost-effectiveness of fertility preservation with $\mathrm{OC}$ compared to expectant management (no-OC) in 25-40year old cancer patients based on estimated gonadotoxicity treatment 5 years after cancer diagnosis. Their model took into account the type of chemotherapy, the potential development of primary ovarian insufficiency after treatment, and effect of age on fertility decline. Low-risk chemotherapy was equated to therapies such as adriamycin, bleomycin, vinblastine, and dacarbazine for management of Hodgkin lymphoma, while high-risk chemotherapy was equated to conditioning chemotherapeutic regimens used for hematopoietic stem cell transplant for leukemia. When comparing OC to no-OC, the maximum improvement in live birth rate was achieved at 37 years old for low-risk chemotherapy (68\% OC vs. $37 \%$ no-OC) and at 27 years old for highrisk chemotherapy (66\% OC vs. $14 \%$ no-OC). The cost per additional live birth in low-risk chemotherapy ranged from $\$ 44,645$ to $\$ 83,424$ with the most cost-effective time period at 37 years old. On the other hand, the cost per additional live birth in high-risk chemotherapy ranged from $\$ 34,194$ to $\$ 75,970$ with the most cost-effective time period at 25 years old. Despite OC being more costly, it is the most cost-effective strategy to improve live birth rates in patients expecting to undergo low or high-risk chemotherapy within 5 years of cancer diagnosis and who are not considering donor oocytes.

\section{Summary}

Women cryopreserving oocytes for medical indications appear to have similar oocyte yields as women undergoing planned $\mathrm{OC}$. The return rate for patients utilizing medical OC is $<10 \%$. There are still limited data on pregnancy and live birth rates from oocytes cryopreserved for medical indications prior to potentially sterilizing therapy.

\section{Planned oocyte cryopreservation}

The ASRM published an evidence-based guideline on planned OC in 2021 but data are limited. These guidelines reviewed the most recent literature and cautioned against planned OC without appropriate counseling regarding the sparsity of data and unknown sequelae [18]. We included eleven primary studies for this review (Table 2). The main areas of focus were live birth rates, effect of age on $\mathrm{OC}$, and number of cryopreserved oocytes needed to obtain a live birth.

\section{Live birth rates in planned oocyte cryopreservation}

Doyle et al. [30] published a retrospective study which found that 128 cycles undergoing autologous embryo transfer (ET) from warmed vitrified oocytes had lower numbers of MII oocytes inseminated (8.0 vs. 10.1, $p=.0002)$ and blastocyst-stage embryo transfers (50.9\% vs. $66.1 \%, p<.001$ ) when compared to 2963 cycles using fresh oocytes. There were similar fertilization rates $(69.5 \%$ vs. $71.7 \%, p>.05)$ and ongoing pregnancy rates (38.6\% vs. $36 \%, p>.05)$, and higher clinical pregnancy rates and clinical pregnancy losses per clinical pregnancy rate in the vitrified oocyte group compared to the fresh oocyte group. One confounding factor in this study is the inclusion of patients undergoing oocyte cryopreservation due to lack of sperm at time of oocyte 


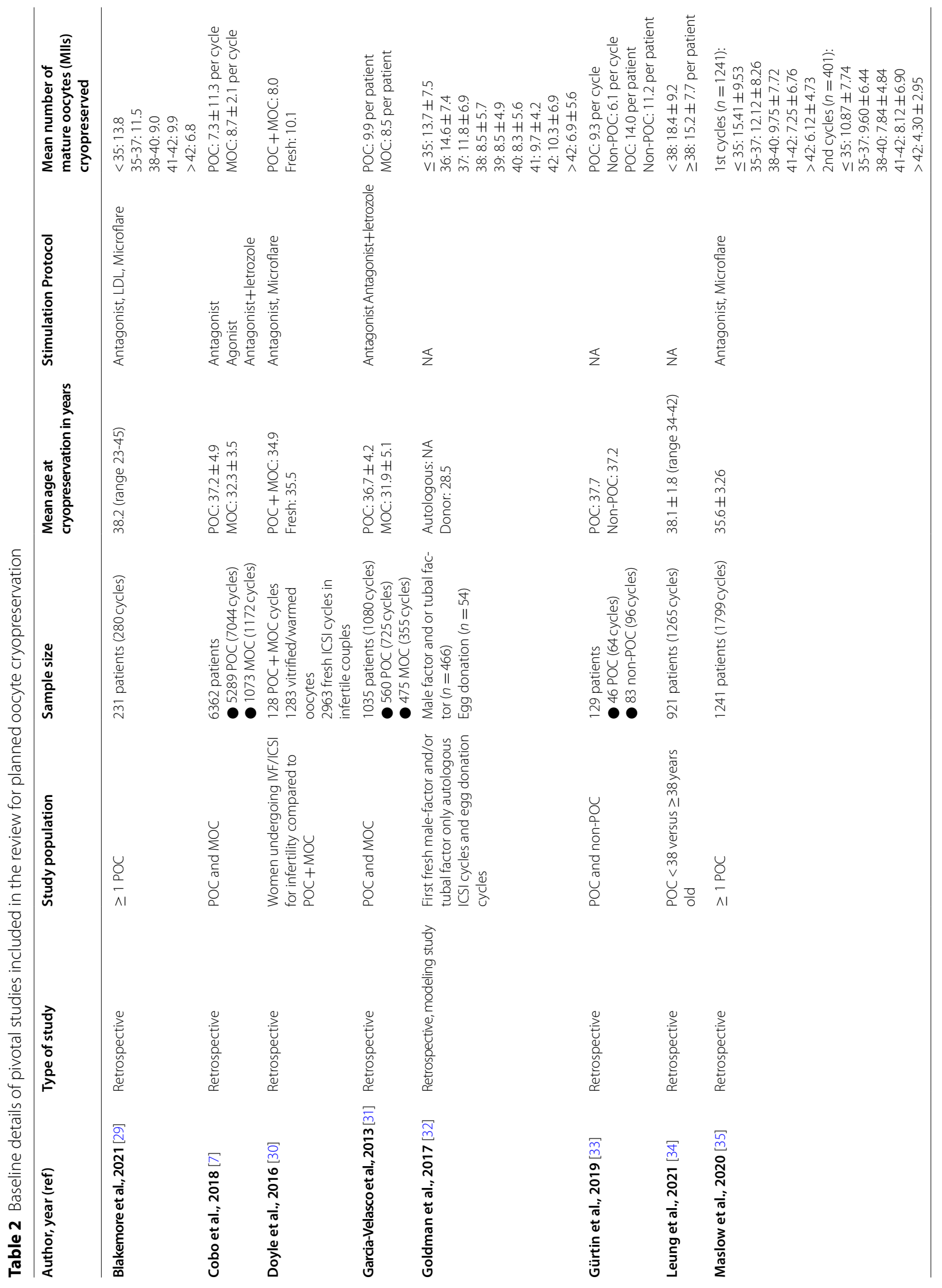




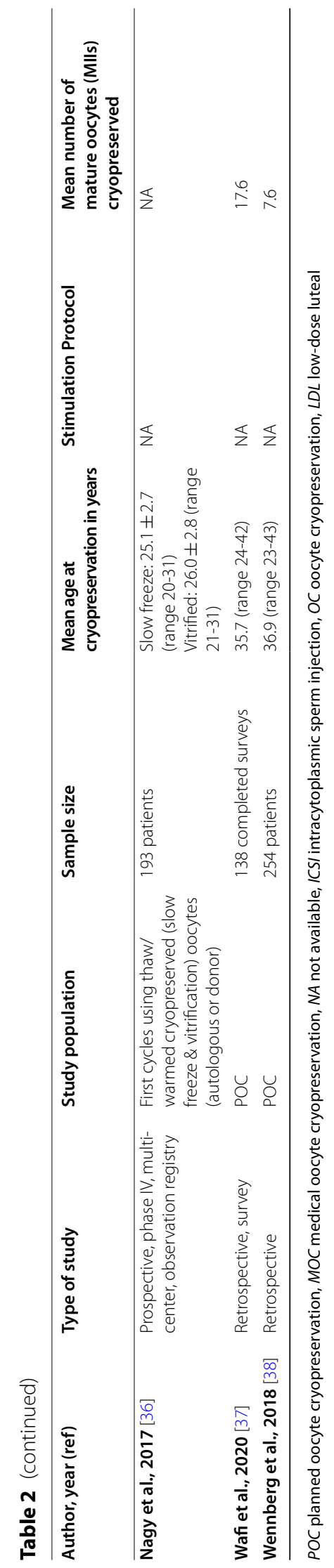


retrieval ( $N=52$ cycles by 51 patients). Although male factor infertility was not found to be statistically significant between the two groups within the analysis, the inclusion of these additional patients who did not strictly meet the definition of planned OC introduces bias into the results of the study for outcomes within patients undergoing planned OC.

The 2018 study by Cobo et al. [7] investigated the indication for fertility preservation related to success in IVF cycles after planned OC and for medical fertility preservation. As previously noted, this was a retrospective, observational multicenter study that included 6362 patients treated from January 2007 to May 2018. Of these, 5289 patients undergoing 7044 cycles, had planned OC. One of the main outcomes of this study was live birth rate (Table 3). The mean age of patients who underwent planned OC was $37.2 \pm 4.9$ with $81.1 \%$ of patients having their oocytes vitrified $>35$ years old. The mean number of mature oocytes retrieved and vitrified per cycle was $9.6 \pm 8.4$ and $7.3 \pm 11.3$, respectively. The survival rate was $83.9 \%$ with an implantation rate of $42.6 \%$. The clinical pregnancy rate per transfer was $50.7 \%$ and ongoing pregnancy rate per transfer was 39.2\%. 115 live births occurred within the planned OC group.

A recent 2021 study by Leung et al. investigated the clinical experience of patients who had undergone planned OC [34]. They performed a retrospective, observational study that included 921 patients (1265 cycles) who underwent planned OC from June 2006 to October 2020 at a single institution within an insurance mandated state. They split their cohort into two groups: $<38$ and $\geq 38$ years old. Mean age of patients included was 36.6 years with the mean age at time of use at $38.1 \pm 1.8$ years (range, 34-42). The mean number of mature oocytes vitrified and mean number of cycles used per patient were $17.1 \pm 8.6$ and $1.4 \pm 0.6$, respectively. There was no significant difference in mean number of oocytes cryopreserved based on age. Oocyte survival rate was $84.9 \%$ after vitrification and $57.1 \%$ after slow-freeze. Fertilization rate was $74 \%$ among both groups. The clinical pregnancy rate per transfer $(54.5 \%$ for $<38$; $39.3 \%$ for $\geq 38$ years old) or per patient who achieved transfer (64.0\% for $<38$; $52.4 \%$ for $\geq 38$ years old) was not different between groups. Also, the live birth rate per transfer

Table 3 Summary of cycle outcomes and utilization of included studies in patient who underwent planned oocyte cryopreservation

\begin{tabular}{|c|c|c|c|c|c|c|}
\hline Author, year (ref) & $\begin{array}{l}\text { Mean duration in } \\
\text { storage (years) }\end{array}$ & Return rate (\%) & Survival rate (\%) & $\begin{array}{l}\text { Mean Fertilization } \\
\text { rate (\%) }\end{array}$ & $\begin{array}{l}\text { Clinical Pregnancy } \\
\text { Rate (\%) }\end{array}$ & $\begin{array}{l}\text { Ongoing Pregnancy/ } \\
\text { Live Birth Rate }\end{array}$ \\
\hline Blakemore et al., 2021 [29] & $\begin{array}{l}<35: 8 \\
35-37: 6.6 \\
38-40: 5.3 \\
41-42: 4.9 \\
>42: 5\end{array}$ & $\begin{array}{l}<35: 40 \\
35-37: 44.1 \\
38-40: 36.3 \\
41-42: 33.3 \\
>42: 25.0\end{array}$ & $\begin{array}{l}<35: 72.8 \\
35-37: 77.0 \\
38-40: 73.7 \\
41-42: 66.6 \\
>42: 78.0\end{array}$ & 68.8 & NA & $\begin{array}{l}\text { Live birth (fresh/eSET) } \\
<35: \text { NA/3 } \\
35-37: 3 / 10 \\
38-40: 3 / 5 \\
41-42: 2 / 0 \\
>42: \text { NA/NA }\end{array}$ \\
\hline Cobo et al., 2018 [7] & $\begin{array}{l}\text { POC: } 2.1 \pm 1.6 \\
\text { MOC: } 4.1 \pm 0.9\end{array}$ & $\begin{array}{l}\text { POC: } 12.1 \\
\text { MOC: } 7.4\end{array}$ & $\begin{array}{l}\text { POC: } 83.9 \\
\text { MOC: } 81.8\end{array}$ & NA & $\begin{array}{l}\text { POC: } 50.7 \text { per transfer } \\
\text { MOC: } 41.4 \text { per transfer }\end{array}$ & $\begin{array}{l}\text { POC: } 39.2 \% \text { per transfer } \\
\text { MOC: } 31.0 \% \text { per transfer }\end{array}$ \\
\hline Doyle et al., 2016 [30] & $\begin{array}{l}\text { Vitrified: } 8.0 \\
\text { Fresh: } 0\end{array}$ & $\begin{array}{l}\text { Vitrified: NA } \\
\text { Fresh: NA }\end{array}$ & $\begin{array}{l}\text { Vitrified: } 86.1 \\
\text { Fresh: NA }\end{array}$ & $\begin{array}{l}\text { Vitrified: } 69.5 \\
\text { Fresh: } 71.7\end{array}$ & $\begin{array}{l}\text { Vitrified: } 54.4 \text { per cycle } \\
\text { Fresh: } 45.1 \text { per cycle }\end{array}$ & $\begin{array}{l}\text { Vitrified: } 38.6 \% \\
\text { Fresh: } 36.0 \%\end{array}$ \\
\hline Fuchs Weizman et al., 2020 [39] & $1.8-4.8$ & $12.1-15$ & $85 \%$ & $66-84 \%$ & $39-84 \%$ & NA \\
\hline Garcia-Velasco et al, 2013 [31] & $\begin{array}{l}\text { POC: } 1.7 \pm 0.6 \\
\text { MOC: NA }\end{array}$ & $\begin{array}{l}\text { POC: } 4.6 \\
\text { MOC: } 0.8\end{array}$ & $\begin{array}{l}\text { POC: } 84.8 \% \\
\text { MOC: NA }\end{array}$ & NA & $\begin{array}{l}\text { POC: } 42.3 \text { per patient } \\
\text { MOC: } 25 \text { per patient }\end{array}$ & $\begin{array}{l}\text { POC: } 30.7 \% \text { per patient } \\
\text { MOC: } 25 \% \text { per patient }\end{array}$ \\
\hline Gürtin et al., 2019 [33] & $\begin{array}{l}\text { POC: } 4.8 \\
\text { Non-POC: } 0.4\end{array}$ & NA & NA & NA & NA & $\begin{array}{l}\text { POC: } 17.4 \% \\
\text { Non-POC: } 22.9 \%\end{array}$ \\
\hline Leung et al., 2021 [34] & $\begin{array}{l}<38: 4.1 \\
\geq 38: 3.2\end{array}$ & $\begin{array}{l}<38: 5.6 \\
\geq 38: 11.9\end{array}$ & $\begin{array}{l}\text { Vitrified: } 84.9 \% \\
\text { Slow: } 57.1 \%\end{array}$ & $\begin{array}{l}<38: 78 \\
\geq 38: 70\end{array}$ & $\begin{array}{l}<38: 54.5 \text { ( } 95 \% \mathrm{Cl} 37.6- \\
\text { 71.5) per transfer } \\
\geq 38: 39.3 \text { ( } 95 \% \mathrm{Cl} 21.2- \\
\text { 57.4) per transfer } \\
<38: 64.0 \text { ( } 95 \% \mathrm{Cl} 45.2- \\
\text { 82.8) per patient } \\
\geq 38: 52.4 \text { ( } 95 \% \mathrm{Cl} 31.0- \\
\text { 73.7) per patient }\end{array}$ & $\begin{array}{l}<38: 48.5 \% \text { ( } 95 \% \text { Cl } 31.4- \\
65.5) \text { per transfer } \\
\geq 38: 28.6 \%(95 \% \mathrm{Cl} 11.8- \\
45.3) \text { per transfer } \\
<38: 56.0 \%(95 \% \mathrm{Cl} 36.5- \\
\text { 75.5) per patient } \\
\geq 38: 38.1 \%(95 \% \mathrm{Cl} 17.3- \\
\text { 58.9) per patient }\end{array}$ \\
\hline Wennberg et al., 2018 [38] & 4.0 & 15 & 78 & 62 & NA & $\begin{array}{l}\text { 36-37: } 56 \% \text { per transfer } \\
\text { 38-39: } 17 \% \text { per transfer } \\
\geq 40: 0 \% \text { per transfer } \\
\text { 36-37: } 63 \% \text { per woman } \\
\text { 38-39: } 26 \% \text { per woman } \\
\geq 40: 0 \% \text { per woman }\end{array}$ \\
\hline
\end{tabular}


(48.5\% for $<38$; $28.6 \%$ for $\geq 38$ years old) or per patient who achieved transfer $(56.0 \%$ for $<38$ year old; $38.1 \%$ for $\geq 38$ years old) was not different between both groups. In addition, the live birth rate among all patients who initiated a thaw/warm cycle $(n=68)$ was $38.9 \%$ for $<38$ years old and $25 \%$ for $\geq 38$ years old. No successful pregnancy occurred in patients who utilized planned OC when $\geq 40$ years old. Some limitations of this study were the inclusion of slow-freeze and vitrification cycles and the limited generalizability given that the study was conducted in an insurance mandated state.

Blakemore et al. conducted a large retrospective study at a single, urban university-affiliated fertility center on patients who underwent $\geq 1$ cycle of planned OC from January 2005 to December 2009 [29]. They analyzed 231 patients ( 280 cycles). Eighty-eight patients (38.1\%) returned to thaw/warm oocytes for intended intracytoplasmic sperm injection (ICSI) and embryo transfer. The mean age of patients returning to thaw/warm oocytes was 43.9 years (range, 38-50years) with a mean duration of oocyte vitrification of 5.9 years (range, $1-12$ years). The mean survival rate post-thaw was $74.2 \%$ (median $73.7 \%$, range $0-100 \%$ ) and the mean fertilization rate was $68.8 \%$; these rates did not differ based on age $(p=0.79)$. There were 41 fresh ET of thawed/warmed oocytes. Thirteen embryo transfers $(31.7 \%)$ with a mean of 3.4 embryos transferred were performed on Day 3. The remaining 28 transfers $(68.3 \%)$ with a mean number of embryos transferred of 1.8 were performed on day 5. Of those who underwent a fresh transfer, $28.2 \%$ of patients achieved a live birth. In addition, 49 patients underwent preimplantation genetic testing for aneuploidy (PGT-A) with the mean of 4.2 embryos being biopsied per patient (range, $0-14$ ). The average euploidy rate was $28.9 \%$. The live birth rate per transfer of an euploid embryo was $66.7 \%$ within the PGT-A group.

A study by Wennberg et al. [38] on planned OC included 254 patients who underwent planned $\mathrm{OC}$ at a private IVF center in Sweden between August 1, 2011 to August 31, 2017. The mean age at first vitrification was 36.9 (range, 23-43) and the mean number of frozen oocytes per patient undergoing $\geq 1$ retrieval was 7.6 (range, 1-37). The mean age of those who utilized their oocytes was 38.7 (range, 36-42) at vitrification and 42.7 (range, 38-45) at thaw/warming. Within the group of patients who returned to use their oocytes, the mean number of mature oocytes banked was 12.8 (range, $1-37$ ) with a $78 \%$ survival rate and $62 \%$ fertilization rate. Live birth rates per transfer were 56,17 , and $0 \%$ at $36-37,38-39$, and $\geq 40$ at age of vitrification, respectively. Alternatively, the live birth rate per patient was 63,26 , and $0 \%$ at $36-37,38-39$, and $\geq 40$ at age of vitrification, respectively. One interesting characteristic of this study worth highlighting is that the local recommendation for patients undergoing planned OC was to obtain $\geq 15-20$ oocytes to achieve an live birth; however, only $18 \%$ of patients were able to achieve this goal suggesting that live births are still possible with lower oocyte yields.

Gürtin et al. performed a retrospective study of planned OC within the UK over a 10 -year time frame [33]. They sought to understand more the characteristics of the group of patients who returned to use their eggs. This analysis included 129 patients stratified into two categories: social egg freeze (SEF) and non-SEF. NonSEF incorporated egg freeze for clinical reasons (e.g., intentional part of IVF treatment or to batch eggs), incidental egg freeze (e.g., no sperm available at time of egg retrieval), and ethical egg freeze (e.g., patients who did not believe in freezing embryos). Ultimately 46 patients underwent 64 cycles in the SEF group (planned OC). The average age at time of freeze was 37.7 years within the SEF group. The success rate, which was defined as both live birth and ongoing pregnancy, was $17.4 \%$ within the SEF group.

A 2020 retrospective questionnaire of patients who had previously undergone planned OC between January 2009 and September 2016 at a single center within the UK also reported on live birth rates [37]. Three hundred and forty-two patients had undergone planned OC during this time frame; however, only 138 completed surveys. The mean age at vitrification was 35.7 (range, 24-42) and the mean follow-up was $4.5 \pm 2.4$ years. The mean number of oocytes cryopreserved was 17.6 (range, 2-64). Sixty-one patients reported they tried to conceive after planned OC. Forty-six percent $(28 / 61)$ of respondents reported that they used their cryopreserved eggs to try to conceive and $46 \%(13 / 28)$ reported having a live birth. The number of ET performed was not reported.

Two smaller observational studies done by GarciaVelasco et al. and Nagy et al. also investigated ongoing pregnancy and live birth rates. Garcia-Velasco et al. reported the results of controlled ovarian hyperstimulation in oncological and non-oncological patients seeking fertility preservation. Five hundred and sixty non-oncological patients were included in the study (mean age $36.7 \pm 4.2$ years); 26 patients returned for thaw/warming of frozen oocytes. Twenty-four fresh embryo transfers were performed (mean number of embryos transferred $1.5 \pm 0.6)$. The clinical pregnancy rate per patient was $42.3 \%$ and the ongoing pregnancy was $30.7 \%$ after a fresh embryo transfer [31]. Fifteen patients underwent a frozen embryo transfer (mean number of embryos transferred $2.3 \pm 0.7$ ), and had a clinical pregnancy rate per patient of $46.6 \%$ and ongoing pregnancy rate per patient of $33.3 \%$ [31]. Nagy et al. reported ART outcomes after autologous oocyte vitrification and thawing in 46 cycles after planned 
OC (mean age 33.9 \pm 3.9 years old (range, 25-43years)) [36]. The mean number of embryos transferred per cycle was $2.7 \pm 1.0$. ( $3.0 \pm 1.0$ day 3 vs. $2.2 \pm 0.7$ day $5 / 6)$. Twelve transfers $(30 \%)$ resulted in a clinical pregnancy and 8 cycles resulted in a live birth (17.4\%). Unfortunately, neither investigation used a control group.

A retrospective cohort study, by Maslow et al., of 1241 patients $(1799$ cycles) at a single, large OC program revealed the likelihood to achieve an estimated live birth rate (eLBR) of 50,60 , and $70 \%$ within $1-2$ cycles of planned OC [35]. Using data from Doyle et al. and Goldman et al. [30, 32], they extrapolated the thresholds for an age-based number of frozen mature oocytes needed to obtain a 50,60 , and $70 \%$ eLBR. The mean number of frozen MIIs based on age is included in Table 2. They found that the main contributors to success in this study were young age, high $\mathrm{AMH}$, high peak estradiol, and low total gonadotropin usage. Sixty-six percent of patients in this study achieved a 50\% eLBR with their first cycle while $51 \%$ of patients achieved an eLBR of $70 \%$ with their first cycle. Having an AMH $>1.995 \mathrm{ng} / \mathrm{dL}$ was predictive of an eLBR of $60 \%$ with their first cycle regardless of age $(p<0.001)$. In addition, patients $<37.5$ years old were more likely to obtain a $60 \%$ eLBR with their first cycle regardless of AMH level $(p<0.001)$. For patients who did not reach a 50\% eLBR during their first cycle, $69.3 \%$ were able to achieve at least $50 \%$ eLBR during their second cycle. Patients $>42$ years old were not able to reach $50 \%$ eLBR within two cycles. Maslow et al. were able to generate a table from their data using age, AMH level, and number of frozen MIIs to predict eLBR (Table 4).

\section{Effect of age at time of oocyte retrieval}

There is currently insufficient data to counsel patients on the optimal age at which to undergo planned OC. Not surprisingly, the few studies available on this subject indicate that younger oocyte age results in higher live birth rates per embryo transfer.

Doyle et al. [30] found that patients who undergo autologous planned OC < 38 years old had higher clinical pregnancy rates per embryo transfer than patients $\geq 38$ years old at time OC $(60.2 \%$ vs. $43.9 \%)$. However, the data included patients who underwent planned $\mathrm{OC}$ as well as patients who underwent $\mathrm{OC}$ for other indications. Nagy et al. [36] found that live birth rates among patients who underwent planned $\mathrm{OC}$ at $<35$ years old were significantly better than patients $\geq 35$ years old (24 (23.8\%) vs. $26(12.0 \%), p<.05)$; however, this study had several limitations because they used the Human Oocyte Preservation Experience registry to enroll patients. Limitations that are accompanied with use of a registry include selection bias due to entry of non-sequential patients, missing data, changes in cryopreservation technique, small sample size, and the inclusion of patients that underwent medically indicated $\mathrm{OC}$ due to lack of sperm at time of egg retrieval. Gürtin et al. [33] also noticed a decline in successful outcomes (defined as live birth rate and ongoing pregnancy rate) with increasing age at time of first vitrification; however, their numbers were too small to measure statistically. Cobo et al. [7] investigated the probability of live birth based on the age at vitrification and found significantly higher cumulative live birth rates for younger patients ( $\leq 35$ years old) compared to older patients $(>35$ years old) $(p<0.0001)$.

Leung et al. [34] reported contrary findings on the significance of age at the time of vitrification. The mean age at time of vitrification was $38.1 \pm 1.8$ years old (range, 34-42 years) with the mean age at oocyte thaw of $41.8 \pm 2.1$ years old. They did not find any statistical significance in cumulative live birth rates between patients who vitrified oocytes at $<38$ and $\geq 38$ years old most likely due to their low return rate $(7.4 \%)$ and subsequent small sample size $(n=46)$ (Table 3$)$. However, no patients who vitrified $\geq 40$ years old had a successful pregnancy.

A systematic review and meta-analysis done by Fuchs Weizman et al. included 43 studies and found that planned $\mathrm{OC}$ is the most cost efficient at 35 years old (assuming a minimum of $60 \%$ utilization) [39, 40]. Another study by Mesen et al. found that planned OC was cost-effective at 37 years old if patients were willing

Table 4 Maslow et al. number of frozen MII oocytes in first retrieval by age and AMH, presented as n; mean \pm SD

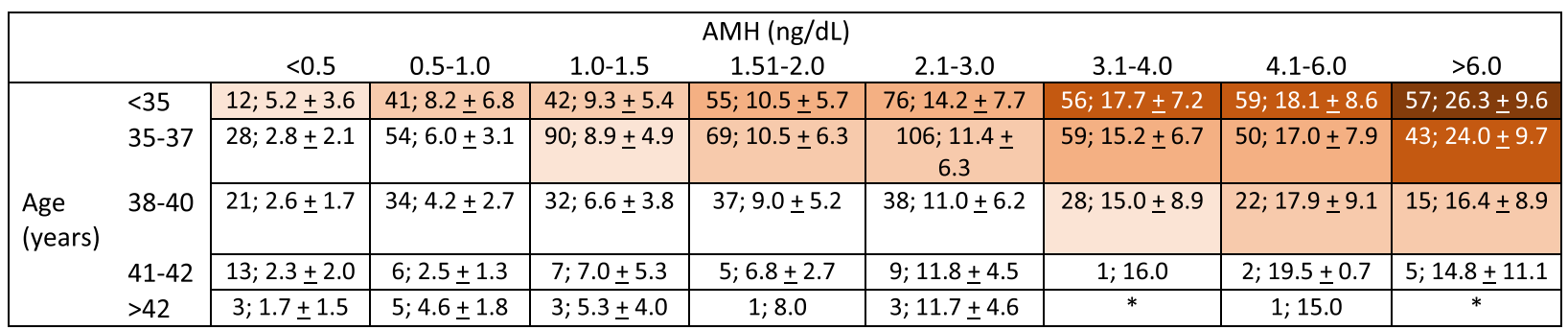

AMH - Anti-Müllerian hormone

Gradations in shading represent: $50 \%, 60 \%, 70 \%, 80 \%$, and $90 \%$ estimated live birth rate. 
to use donor sperm [41]. Mesen et al. used two separate models to perform a cost-effectiveness analysis based on the time a patient makes the decision to undergo planned OC to attempting conception 3, 5, or 7 years later. Model A compared $\mathrm{OC}$ to no-OC and assumed that a patient would only attempt to conceive after marriage. Model $\mathrm{B}$ compared $\mathrm{OC}$ to no-OC and assumed that a patient would attempt to conceive regardless of marital status (willing to use spouse, partner, or donor sperm). Model A patients who used $\mathrm{OC}$ were not found to have a substantially better live birth rate $(<10 \%)$ when compared to Model A patients who did not undergo planned OC and were awaiting marital status. Model B was found to be the most cost-effective if planned OC was performed at 37 years old compared to other Model B patients who did not undergo planned $\mathrm{OC}$ and were not awaiting marital status. The probability of live birth rate was $29.7 \%$ higher (51.6\% live birth rate for OC vs. $21.9 \%$ live birth rate for no-OC) and approximately $\$ 9000$ more expensive in cost (\$19,493 with OC vs. $\$ 10,943$ without OC) [41].

Another study by Devine et al. found that it was more cost-effective for women who were $>38$ years old to defer planned OC and to undergo two cycles of IVF [42]. Devine et al. used theoretical models to map out three different strategies for 35 year old patients who deferred childbearing for personal reasons until 40 years old. Strategy $1(\mathrm{OC})$ consisted of undergoing 1.2 cycles of planned OC (mean number required to obtain $16 \mathrm{MII}$ oocytes at 35 years old), attempt spontaneous conception at 40 years old for 6 months, 2 oocyte thaw cycles using stored oocytes if no live birth. Strategy 2 (OC/IVF) consisted of undergoing 1.2 cycles of planned OC (mean number required to obtain $16 \mathrm{MII}$ oocytes at 35 years old), attempt spontaneous conception at 40 years old for 6 months, 2 fresh autologous ART cycles if no live birth, 2 oocyte thaw cycles using stored oocytes if still no live birth. Strategy 3 (no-OC) consisted of no OC at 35 years old, attempt spontaneous conception at 40 years old for 6 months, 2 fresh autologous ART cycles if no live birth. Strategy 1 was found to be the most cost-effective in achieving live birth until 38 years of age when Strategy 3 was most cost-effective, thereby presuming it may be more cost-effective for patients who are considering to undergo planned $\mathrm{OC}$ at $\geq 38$ years old to try spontaneous conception for 6 months then if not pregnant to undergo 2 fresh autologous ART cycles.

\section{How many oocytes should be frozen to have a reasonable chance at live birth?}

Few studies have evaluated the number of cryopreserved mature oocytes needed to achieve a live birth. Based on their data, Doyle et al. estimated that to achieve a $70 \%$ chance of one live birth in their cohort of 128 autologous thawed/warmed treatment cycles, patients 30-34years old would need 14 MIIs, 35-37 years old would need 15 MIIs, and 38-40 years old would need 26 MIIs [30]. Cobo et al. found that 10 or 15 vitrified oocytes yielded success rates of $42.8 \%$ (95\% $\mathrm{CI}=31.7-53.9)$ and $69.85 \%(95 \%$ $\mathrm{CI}=57.4-82.2)$ for cumulative live birth rates, respectively. For women $\leq 35$ years old, the cumulative live birth rate plateaued at 24 vitrified oocytes with a $94.4 \%$ [95\% $\mathrm{CI}=84.3-100.4]$ cumulative live birth rate.

Goldman et al. conducted a modeling study from a retrospective analysis of their academic infertility center data. They included 520 fresh autologous cycles using ICSI in patients with only male factor infertility, tubal factor infertility, and/or egg donation. Patients with evidence of hydrosalpinx, diminished ovarian reserve, or utilizing preimplantation genetic testing were excluded from the study. Statistical models that included the percentage of mature eggs expected to survive warming, fertilized and become blastocysts were developed to calculate blastulation potential per mature oocyte retrieved. The probability of any one blastocyst being euploid was calculated using age-specific data from Reprogenetics. They included data of 520 patients and found that patients 34,37 , and 42 years old would have to freeze 10 , 20 , and 61 MIIs, respectively, to achieve a $75 \%$ chance of having one or more live birth [32]. The data were used to develop a phone app that can be used to calculate expected live birth rates at different ages based on the number of MII eggs frozen [43].

Nagy et al. employed a similar concept using autologous planned OC treatment cycles and found that patients $<35$ years old required 38.8 MIIs to achieve one live birth while patients $\geq 35$ years old required 77 MIIs [36]. However, their data included a small sample size for planned $\mathrm{OC}$, nonelective indications for cryopreservation, and conclusions based on calculated values and not actual data.

The ASRM practice committee concluded that there is insufficient evidence to counsel patients on the ideal number of oocytes required to achieve one live birth with planned OC [18].

\section{Utilization of cryopreserved oocytes}

The 2018 study by Cobo et al. also investigated utilization of cryopreserved oocytes [7]. They noticed an increase in the utilization of planned OC from 4 to $22 \%$ over the course of the study, from 2007 to 2018. The return rate for patients who used planned OC was $12.1 \%$ with a mean storage time of $2.1 \pm 1.6$ years.

Blakemore et al. studied a 10-15 year follow-up period after planned OC [29]. Of the 231 patients enrolled only $88(38.1 \%)$ of patients underwent thaw/warming while $136(58.9 \%)$ did not use and 7 (3.0\%) transported oocytes to another center with unknown outcome. Rates 
of utilization did not differ by age group. They also found that of the 88 patients who utilized their vitrified oocytes most returned to thaw/warm oocytes with a partner $(n=55,62.5 \%)$ compared to donor sperm $(n=33,37.5 \%)$ and this did not differ based on age. Fuchs Weizman et al. also evaluated the utilization of oocytes after planned OC in a systematic review and found that $12.1-15 \%$ of oocytes were used 22-58 months after freezing [39].

A recent 2021 study by Leung et al. found a lower return rate of $7.4 \%$ over a 14 year time-frame [34]. Patients who were $\geq 38$ years old at time of vitrification were more likely to utilize their cryopreserved oocytes (11.9\%) compared to patients $<38$ years old $(5.6 \%)(p<0.0009)$. The average storage duration was $3.7 \pm 1.7$ years. Most patients who returned to thaw/warm their oocytes had a partner to provide sperm, which sperm source (e.g., partner vs. donor) was not statistically significant between groups. The authors did identify that the mean age of first freeze is steadily declining within recent years; however, individuals who have used their frozen eggs have been in older age groups ( $\geq 38$ years old).

Wafi et al. [37] found that $65 \%$ of survey respondents who had undergone planned OC anticipated using their oocytes in the future, and the maximum age considered for use of frozen eggs was 45.2 years old. $98 \%$ of respondents reported that they would recommend planned OC to others. Interestingly, when asked about what they would do about unused cryopreserved oocytes, $62 \%$ reported donating them to research and $14 \%$ would donate them to other patients.

\section{Obstetrical \& Perinatal Outcomes}

There are limited studies on the obstetrical and perinatal outcomes of babies born after planned OC. The limited amount of evidence available conclude that neonatal outcomes are similar between fresh and cryopreserved oocytes in infertile patients [31, 44-47].

\section{Effects of BMI, ethnicity, and smoking on planned OC}

There are currently no data available investigating the effects of demographics on planned OC (e.g., ethnicity, body mass index, smoking). Future studies are needed to uncover the potential impact of these factors that may ultimately affect decision-making and counseling.

\section{Summary}

Planned $\mathrm{OC}$ is a valuable option for patients wishing to attempt to preserve their future fertility. Available data are sparse, so how many cryopreserved oocytes lead to a high likelihood of live birth is still not concretely defined. As expected, due to the impact of age on ovarian reserve and oocyte aneuploidy, patients who undergo planned $\mathrm{OC}$ at younger ages have the highest likelihood and potentially require the least number of cycles. There does not appear to be an increase in congenital anomalies with use of planned OC.

Thus far it appears that most patients who undergo planned $\mathrm{OC}$ do not return to use them, regardless of age at time of preservation [29]. Surveys of patients who have undergone planned $\mathrm{OC}$ and achieved natural conception do not regret undergoing planned OC [16]. There is currently a strong push by Society for Assisted Reproductive Technology (SART) and ASRM to monitor and report planned OC cycles to accrue more data and help guide management options for future patients [18].

\section{Conclusion}

It is too early to draw any final conclusions on outcomes of OC in medical OC and planned OC; however, preliminary data supports that utilization of $\mathrm{OC}$ in both groups result in preservation of fertility and subsequent live births in patients who return to use their cryopreserved eggs. Higher oocyte yield, with fewer ovarian stimulation cycles, and higher live birth rates are seen in patients who seek $\mathrm{OC}$ at younger ages, reinforcing the importance of age on fertility preservation. There is currently a low utilization rate of cryopreserved oocytes within each group. Less than $10 \%$ of medical OC patients and approximately $40 \%$ of planned OC patients have returned to use their cryopreserved oocytes, which limits future studies on clinical outcomes. More studies are needed in medical $O C$ and planned $O C$ to help guide counseling and decision-making in patients seeking these services.

\section{Abbreviations \\ ACOG : American College of Obstetricians and Gynecologist; ART : Assisted Reproductive Technology; ASRM : American Society of Reproductive Medicine; $\mathrm{BMI}$ : Body mass index; eLBR: Estimated live birth rate; $\mathrm{ET}$ : Embryo transfer; ICSI : Intracytoplasmic sperm injection; IVF : In vitro fertilization; MII : Meta- phase II/mature oocyte; OC : Oocyte cryopreservation; PGT-A : Preimplantation genetic testing for aneuploidy; SART CORS : Society for Assisted Reproductive Technology Clinical Outcomes Reporting System; SEF : Social egg freeze.}

\section{Acknowledgements \\ None.}

Authors' contributions

Z.W. was responsible for manuscript writing and editing. A.L. was responsible for manuscript editing. E.G. was responsible for supervision and manuscript editing. The authors read and approved the final manuscript.

\section{Funding}

None.

Availability of data and materials

Data sharing not applicable to this article as no datasets were generated or analyzed during the current study.

\section{Declarations}

Ethics approval and consent to participate

This study did not include human participants and therefore IRB approval was waived. 


\section{Consent for publication}

Not applicable.

\section{Competing interests}

None.

Received: 14 October 2021 Accepted: 21 December 2021

Published online: 07 January 2022

\section{References}

1. Chen C. Pregnancy after human oocyte cryopreservation. Lancet. 1986;327(8486):884-6 [cited 2021 Aug 20]. Available from: https://pubmed.ncbi.nlm.nih.gov/2870356/.

2. Rienzi L, Gracia C, Maggiulli R, Labarbera AR, Kaser DJ, Ubaldi FM, et al. Oocyte, embryo and blastocyst cryopreservation in art: systematic review and meta-analysis comparing slow-freezing versus vitrification to produce evidence for the development of global guidance. Hum Reprod Update. 2017;23(2):139-55 [cited 2021 Aug 25]. Available from: https:// pubmed.ncbi.nlm.nih.gov/27827818/.

3. Pouget O, Scalici E, Hoa-Ferrieres A, Deutsch-Bringer S, Gala A, Dechaud $\mathrm{H}$, et al. Comparaison des issues de transferts d'embryons congelés au Stade blastocyste selon la technique de congélation et le type de préparation endométriale. Gynecol Obstet Fertil. 2015;43(3):219-24 [cited 2021 Aug 25]. Available from: https://pubmed.ncbi.nlm.nih.gov/25316444/.

4. Oocyte cryopreservation. Obstet Gynecol. 2014;123(1):221-2 [cited 2021 Aug 20]. Available from: https://journals.Iww.com/greenjournal/Fulltext/ 2014/01000/Committee_Opinion_No_584_Oocyte_Cryopreservation. 39.aspx.

5. SART. National summary report. Soc Assisted Reprod Technol. 2021; [cited 2019 Feb 13]. Available from: https://www.sartcorsonline.com/rptCSR_ PublicMultYear.aspx?reporting Year $=2018$.

6. Daar J, Benward J, Collins L, Davis J, Davis O, Francis L, et al. Planned oocyte cryopreservation for women seeking to preserve future reproductive potential: an ethics committee opinion. Fertil Steril. 2018;110(6):1022-8 [cited 2021 Aug 20], Available from: https://pubmed. ncbi.nlm.nih.gov/30396539/.

7. Cobo A, García-Velasco J, Domingo J, Pellicer A, Remohí J. Elective and Onco-fertility preservation: factors related to IVF outcomes. Hum Reprod. 2018;33(12):2222-31 [cited 2021 Aug 21]. Available from: https://pubmed.ncbi.nlm.nih.gov/30383235/.

8. Hodes-Wertz B, Druckenmiller S, Smith M, Noyes N. What do reproductive-age women who undergo oocyte cryopreservation think about the process as a means to preserve fertility? Fertil Steril. 2013;100(5) [cited 2021 Aug 21]. Available from: https://pubmed.ncbi.nlm.nih.gov/23953 $326 /$.

9. Tozzo P, Fassina A, Nespeca P, Spigarolo G, Caenazzo L. Understanding social oocyte freezing in Italy: a scoping survey on university female students' awareness and attitudes. Life Sci Soc Policy. 2019;15(1) [cited 2021 Aug 26]. Available from: https://pubmed.ncbi.nlm.nih.gov/31049743/.

10. Daniluk JC, Koert E, Cheung A. Childless women's knowledge of fertility and assisted human reproduction: identifying the gaps. Fertil Steril. 2012;97(2):420-6 [cited 2021 Aug 26]. Available from: https://pubmed. ncbi.n/m.nih.gov/22192349/.

11. Lampic C, Svanberg AS, Karlström P, Tydén T. Fertility awareness, intentions concerning childbearing, and attitudes towards parenthood among female and male academics. Hum Reprod. 2006;21(2):558-64 [cited 2021 Aug 26]. Available from: https://pubmed.ncbi.nlm.nih.gov/ 16293651/.

12. Peterson BD, Pirritano M, Tucker L, Lampic C. Fertility awareness and parenting attitudes among American male and female undergraduate university students. Hum Reprod. 2012;27(5):1375-82 [cited 2021 Aug 26]. Available from: https://pubmed.ncbi.nlm.nih.gov/22407698/.

13. Kahlor LA, Mackert M. Perceptions of infertility information and support sources among female patients who access the internet. Fertil Steril. 2009;91(1):83-90 [cited 2021 Aug 21]. Available from: https://pubmed. ncbi.nlm.nih.gov/18243181/.

14. Fritz R, Klugman S, Lieman $H$, Schulkin J, Taouk L, Castleberry N, et al. Counseling patients on reproductive aging and elective fertility preservation-a survey of obstetricians and gynecologists' experience, approach, and knowledge. J Assist Reprod Genet. 2018;35(9):1613-21 [cited 2021 Aug 21]. Available from: https://pubmed.ncbi.nlm.nih.gov/ 30073435/.

15. Baldwin K, Culley L, Hudson N, Mitchell H, Lavery S. Oocyte cryopreservation for social reasons: demographic profile and disposal intentions of UK users. Reprod BioMed Online. 2015;31(2):239-45 [cited 2021 Aug 21]. Available from: https://pubmed.ncbi.nlm.nih.gov/26099440/.

16. Jones BP, Kasaven L, L'Heveder A, Jalmbrant M, Green J, Makki M, et al. Perceptions, outcomes, and regret following social egg freezing in the UK; a cross-sectional survey. Acta Obstet Gynecol Scand. 2020;99(3):324-32 [cited 2021 Aug 21]. Available from: https://pubmed.ncbi.nlm.nih.gov/31667820/.

17. Inhorn MC, Birenbaum-Carmeli D, Westphal LM, Doyle J, Gleicher N, Meirow D, et al. Ten pathways to elective egg freezing: a binational analysis. J Assist Reprod Genet. 2018;35(11):2003-11 [cited 2021 Aug 21]. Available from: https://pubmed.ncbi.nlm.nih.gov/30074130/.

18. Evidence-based outcomes after oocyte cryopreservation for donor oocyte in vitro fertilization and planned oocyte cryopreservation: a guideline. Fertil Steril. 2021;116(1):36-47 [cited 2021 Aug 20]. Available from: https://pubmed.ncbi.nlm.nih.gov/34148587/.

19. Letourneau JM, Melisko ME, Cedars MI, Rosen MP. A changing perspective: improving access to fertility preservation. Nat Rev Clin Oncol. 2011;8(1):56-60 [cited 2021 Aug 21]. Available from: https://pubmed. ncbi.nlm.nih.gov/20736926/.

20. Kawwass JF, Shandley LM, Boulet SL, Hipp HS. Oncologic oocyte cryopreservation: national comparison of fertility preservation between women with and without cancer. J Assist Reprod Genet. 2020;37(4):883-90 [cited 2021 Aug 21]. Available from: /pmc/articles/ PMC7183023/.

21. Marklund A, Eloranta S, Wikander I, Kitlinski ML, Lood M, Nedstrand E, et al. Efficacy and safety of controlled ovarian stimulation using $\mathrm{GnRH}$ antagonist protocols for emergency fertility preservation in young women with breast cancer - a prospective nationwide Swedish multicenter study. Hum Reprod. 2020;35(4):929-38 [cited 2021 Nov 28]. Available from: /pmc/articles/PMC7192532/.

22. De Moraes CC, Marinho VFW, Campos ALM, De Souza GJ, De Sousa Xavier ÉB, Caetano JPJ, et al. Oocyte cryopreservation for future fertility: comparison of ovarian response between cancer and non-cancer patients. J Bras Reprod Assist. 2019;23(2):91-8 [cited 2021 Nov 28]. Available from: /pmc/articles/PMC6501752/.

23. Pereira N, Kelly AG, Stone LD, Witzke JD, Lekovich JP, Elias RT, et al. Gonadotropin-releasing hormone agonist trigger increases the number of oocytes and embryos available for cryopreservation in cancer patients undergoing ovarian stimulation for fertility preservation. Fertil Steril. 2017;108(3):532-8 [cited 2021 Nov 28]. Available from: https:// pubmed.ncbi.nlm.nih.gov/28865552/.

24. Quinn MM, Cakmak H, Letourneau JM, Cedars MI, Rosen MP. Response to ovarian stimulation is not impacted by a breast cancer diagnosis. Hum Reprod. 2017;32(3):568-74 [cited 2021 Aug 21]. Available from: https://pubmed.ncbi.nlm.nih.gov/28122888/.

25. Schon SB, Shapiro M, Gracia C, Senapati S. Medical and elective fertility preservation: impact of removal of the experimental label from oocyte cryopreservation. J Assist Reprod Genet. 2017;34(9):1207-15 [cited 2021 Nov 28]. Available from: https://pubmed.ncbi.nlm.nih.gov/28656 539/.

26. Specchia C, Baggiani A, Immediata V, Ronchetti C, Cesana A, Smeraldi $A$, et al. Oocyte cryopreservation in oncological patients: eighteen years experience of a tertiary care referral center. Front Endocrinol (Lausanne). 2019;10:600 [cited 2021 Nov 21]. Available from: /pmc/ articles/PMC6733913/.

27. Turan V, Quinn MM, Dayioglu N, Rosen MP, Oktay K. The impact of malignancy on response to ovarian stimulation for fertility preservation: a meta-analysis. Fertil Steril. 2018;110(7):1347-55 [cited 2021 Aug 21]. Available from: https://pubmed.ncbi.nlm.nih.gov/30503134/.

28. Lyttle Schumacher B, Grover N, Mesen T, Steiner A, Mersereau J. Modeling of live-birth rates and cost-effectiveness of oocyte cryopreservation for cancer patients prior to high- a nd low-risk gonadotoxic chemotherapy. Hum Reprod. 2017;32(10):2049-55 [cited 2021 Nov 28]. Available from: /pmc/articles/PMC5850300/. 
29. Blakemore JK, Grifo JA, DeVore SM, Hodes-Wertz B, Berkeley AS. Planned oocyte cryopreservation-10-15-year follow-up: return rates and cycle outcomes. Fertil Steril. 2021;115(6):1511-20 [cited 2021 Aug 20]. Available from: https://pubmed.ncbi.nlm.nih.gov/33712289/.

30. Doyle JO, Richter KS, Lim J, Stillman RJ, Graham JR, Tucker MJ. Successful elective and medically indicated oocyte vitrification and warming for autologous in vitro fertilization, with predicted birth probabilities for fertility preservation according to number of cryopreserved oocytes and age at retrieval. Fertil Steril. 2016;105(2):459-466.e2 [cited 2021 Aug 20]. Available from: https://pubmed.ncbi.nlm.nih.gov/26604 065/.

31. Garcia-Velasco JA, Domingo J, Cobo A, Martínez M, Carmona L. Pellicer a. five years' experience using oocyte vitrification to preserve fertility for medical and nonmedical indications. Fertil Steril. 2013:99(7):1994-9 [cited 2021 Aug 20]. Available from: https://pubmed.ncbi.nlm.nih.gov/ 23465707/.

32. Goldman RH, Racowsky C, Farland LV, Munné S, Ribustello L, Fox JH. Predicting the likelihood of live birth for elective oocyte cryopreservation: a counseling tool for physicians and patients. Hum Reprod. 2017:32(4):853-9 [cited 2021 Aug 20]. Available from: https://pubmed. ncbi.nlm.nih.gov/28166330/.

33. Gürtin ZB, Morgan L, O'Rourke D, Wang J, Ahuja K. For whom the egg thaws: insights from an analysis of 10 years of frozen egg thaw data from two UK clinics, 2008-2017. J Assist Reprod Genet. 2019;36(6):1069-80 [cited 2021 Nov 18]. Available from: /pmc/articles/ PMC6603120/.

34. Leung AQ, Baker K, Vaughan D, Shah JS, Korkidakis A, Ryley DA, et al Clinical outcomes and utilization from over a decade of planned oocyte cryopreservation. Reprod BioMed Online. 2021;43(4):671-9 [cited 2021 Nov 18]. Available from: https://pubmed.ncbi.nlm.nih.gov/ 34474973/.

35. Maslow BSL, Guarnaccia MM, Ramirez L, Klein JU. Likelihood of achieving a $50,60 \%$, or $70 \%$ estimated live birth rate threshold with 1 or 2 cycles of planned oocyte cryopreservation. J Assist Reprod Genet. 2020;37(7):1637-43 [cited 2021 Aug 20]. Available from: https://pubmed.ncbi.nlm.nih.gov/32418136/.

36. Nagy ZP, Anderson RE, Feinberg EC, Hayward B, Mahony MC. The human oocyte preservation experience (HOPE) registry: evaluation of cryopreservation techniques and oocyte source on outcomes. Reprod Biol Endocrinol. 2017;15(1) [cited 2021 Aug 20]. Available from: https:// pubmed.ncbi.nlm.nih.gov/28173814/.

37. Wafi A, Nekkebroeck J, Blockeel C, De Munck N, Tournaye H, De Vos M. A follow-up survey on the reproductive intentions and experiences of women undergoing planned oocyte cryopreservation. Reprod BioMed Online. 2020;40(2):207-14 [cited 2021 Nov 18]. Available from: https:// pubmed.ncbi.nlm.nih.gov/31983546/.

38. Wennberg AL, Schildauer K, Brännström M. Elective oocyte freezing for nonmedical reasons: a 6-year report on utilization and in vitro fertilization results from a Swedish center. Acta Obstet Gynecol Scand. 2019;98(11):1429-34 [cited 2021 Nov 18]. Available from: https://onlin elibrary.wiley.com/doi/full/10.1111/aogs.13673.

39. Fuchs Weizman N, Baram S, Montbriand J, Librach CL. Planned oocyte cryopreservation (planned $\mathrm{OC}$ ): systematic review and meta-analysis of cost-efficiency and patients' perspective. BJOG An Int J Obstet Gynaecol. 2021;128(6):950-62 [cited 2021 Aug 20]. Available from: https:// pubmed.ncbi.nlm.nih.gov/33021076/.

40. Van Loendersloot LL, Moolenaar LM, BWJ M, Repping S, Van Der Veen F, Goddijn M. Expanding reproductive lifespan: a cost-effectiveness study on oocyte freezing. Hum Reprod. 2011;26(11):3054-60 [cited 2021 Aug 20]. Available from: https://pubmed.ncbi.nlm.nih.gov/21896545/.

41. Mesen TB, Mersereau JE, Kane JB, Steiner AZ. Optimal timing for elective egg freezing. Fertil Steril. 2015;103(6):1551-1556.e4 [cited 2021 Aug 20]. Available from: https://pubmed.ncbi.nlm.nih.gov/25881876/.

42. Devine K, Mumford SL, Goldman KN, Hodes-Wertz B, Druckenmiller S, Propst AM, et al. Baby budgeting: oocyte cryopreservation in women delaying reproduction can reduce cost per live birth. Fertil Steril. 2015;103(6):1446-1453.e2 [cited 2021 Aug 20]. Available from: https:// pubmed.ncbi.nlm.nih.gov/25813281/.

43. Goldman, R. H., Fox JH. BWH Egg Freezing Counseling Tool (EFCT) MDCalc. [cited 2021 Nov 28]. Available from: https://www.mdcalc.com/ bwh-egg-freezing-counseling-tool-efct
44. Chamayou S, Sicali M, Alecci C, Ragolia C, Liprino A, Nibali D, et al. The accumulation of vitrified oocytes is a strategy to increase the number of euploid available blastocysts for transfer after preimplantation genetic testing. J Assist Reprod Genet. 2017;34(4):479-86 [cited 2021 Aug 20]. Available from: https://pubmed.ncbi.nlm.nih.gov/28070710/.

45. Anzola AB, Pauly V, Geoffroy-Siraudin C, Gervoise-Boyer MJ, Montjean $D$, Boyer $P$. The first 50 live births after autologous oocyte vitrification in France. J Assist Reprod Genet. 2015;32(12):1781-7 [cited 2021 Aug 20]. Available from: https://pubmed.ncbi.nlm.nih.gov/26519416/.

46. Noyes N, Porcu E, Borini A. Over 900 oocyte cryopreservation babies born with no apparent increase in congenital anomalies. Reprod BioMed Online. 2009;18(6):769-76 [cited 2021 Aug 20]. Available from: https://pubmed.ncbi.nlm.nih.gov/19490780/.

47. Siano L, Engmann L, Nulsen J, Benadiva C. A prospective pilot study comparing fertilization and embryo development between fresh and vitrified sibling oocytes. Conn Med. 2013;77:211-7.

\section{Publisher's Note}

Springer Nature remains neutral with regard to jurisdictional claims in published maps and institutional affiliations. 